\title{
Research Article \\ Grüss-Type Bounds for the Covariance of Transformed Random Variables
}

\author{
Martín Egozcue, ${ }^{1,2}$ Luis Fuentes García, ${ }^{3}$ Wing-Keung Wong, ${ }^{4}$ \\ and Ričardas Zitikis ${ }^{5}$ \\ ${ }^{1}$ Department of Economics, University of Montevideo, Montevideo 11600, Uruguay \\ ${ }^{2}$ Accounting and Finance Department, Norte Construcciones, Punta del Este 20100, Uruguay \\ ${ }^{3}$ Departamento de Métodos Matemáticos e de Representacion, Escola Técnica Superior de Enxeñeiros \\ de Camiños, Canais e Portos, Universidade da Coruña, 15001 A Coruña, Spain \\ ${ }^{4}$ Department of Economics, Institute for Computational Mathematics, Hong Kong Baptist University, \\ Kowloon Tong, Hong Kong \\ ${ }^{5}$ Department of Statistical and Actuarial Sciences, University of Western Ontario, London, \\ ON, Canada N6A 5B7
}

Correspondence should be addressed to Ričardas Zitikis, zitikis@stats.uwo.ca

Received 9 November 2009; Revised 28 February 2010; Accepted 16 March 2010

Academic Editor: Soo Hak Sung

Copyright (c) 2010 Martín Egozcue et al. This is an open access article distributed under the Creative Commons Attribution License, which permits unrestricted use, distribution, and reproduction in any medium, provided the original work is properly cited.

A number of problems in Economics, Finance, Information Theory, Insurance, and generally in decision making under uncertainty rely on estimates of the covariance between (transformed) random variables, which can, for example, be losses, risks, incomes, financial returns, and so forth. Several avenues relying on inequalities for analyzing the covariance are available in the literature, bearing the names of Chebyshev, Grüss, Hoeffding, Kantorovich, and others. In the present paper we sharpen the upper bound of a Grüss-type covariance inequality by incorporating a notion of quadrant dependence between random variables and also utilizing the idea of constraining the means of the random variables.

\section{Introduction}

Analyzing and estimating covariances between random variables is an important and interesting problem with manifold applications to Economics, Finance, Actuarial Science, Engineering, Statistics, and other areas (see, e.g., Egozcue et al. [1], Furman and Zitikis [2-5], Zitikis [6], and references therein). Well-known covariance inequalities include those of Chebyshev and Grüss (see, e.g., Dragomir [7] and references therein). There are many interesting applications of Grüss's inequality in areas such as Computer Science, Engineering, and Information Theory. In particular, the inequality has been actively investigated in the context of Guessing Theory, and we refer to Dragomir and Agarwal [8], Dragomir and Diamond [9], Izumino and Pečarić [10], Izumino et al. [11], and references therein. 
Motivated by an open problem posed by Zitikis [6] concerning Grüss's bound in the context of dependent random variables, in the present paper we offer a tighter Grüss-type bound for the covariance of two transformed random variables by incorporating a notion of quadrant dependence and also utilizing the idea of constraining the means of the random variables. To see how this problem arises in the context of insurance and financial pricing, we next present an illustrative example. For further details and references on the topic, we refer to Furman and Zitikis [2-5].

Let $X$ be an insurance or financial risk, which from the mathematical point of view is just a random variable. In this context, the expectation $\mathrm{E}[\mathrm{X}]$ is called the net premium. The insurer, wishing to remain solvent, naturally charges a premium larger than $\mathrm{E}[\mathrm{X}]$. As demonstrated by Furman and Zitikis $[2,4]$, many insurance premiums can be written in the form

$$
\pi_{w}[X]=\frac{\mathbf{E}[X w(X)]}{\mathbf{E}[w(X)]}
$$

where $w$ is a nonnegative function, called the weight function, and so $\pi_{w}[X]$ is called the weighted premium. It is well known (Lehmann [12]) that if the weight function $w$ is nondecreasing, then the inequality $\pi_{w}[X] \geq \mathrm{E}[\mathrm{X}]$ holds, which is called the nonnegative loading property in insurance. (Note that when $w(x) \equiv 1$, then $\pi_{w}[X]=\mathrm{E}[X]$.) The weighted premium $\pi_{w}[X]$ can be written as follows:

$$
\pi_{w}[X]=\mathbf{E}[X]+\frac{\operatorname{Cov}[X, w(X)]}{\mathrm{E}[w(X)]}
$$

with the ratio on the right-hand side known as the loading. The loading is a nonnegative quantity because the weight function $w$ is non-decreasing. We want to know the magnitude of the loading, given what we might know or guess about the weight function $w$ and the random variable $X$. Solving this problem naturally leads to bounding the covariance $\operatorname{Cov}[X, w(X)]$.

More generally, as noted by Furman and Zitikis [2,4], we may wish to work with the (doubly) weighted premium

$$
\pi_{v, w}[X]=\frac{\mathbf{E}[v(X) w(X)]}{\mathbf{E}[w(X)]}
$$

The latter premium leads to the covariance $\operatorname{Cov}[v(X), w(X)]$. Finally, in the more general context of capital allocations, the weighted premiums are extended into weighted capital allocations (Furman and Zitikis [3-5]), which are

$$
\begin{aligned}
\pi_{v, w}[X, Y] & =\frac{\mathrm{E}[v(X) w(Y)]}{\mathrm{E}[w(Y)]} \\
& =\mathrm{E}[v(X)]+\frac{\operatorname{Cov}[v(X), w(Y)]}{\mathrm{E}[w(Y)]},
\end{aligned}
$$

where the random variable $Y$ can be viewed, for example, as the return on an entire portfolio and $X$ as the return on an asset in the portfolio. In Economics, $E[v(X)]$ is known as the 
expected utility, or the expected valuation, depending on a context. The 'loading' ratio on the right-hand side of (1.4) can be negative, zero, or positive, depending on the dependence structure between the random variables $X$ and $Y$, and also depending on the monotonicity of functions $v$ and $w$. Our research in this paper is devoted to understanding the covariance $\operatorname{Cov}[v(X), w(Y)]$ and especially its magnitude, depending on the information that might be available to the researcher and/or decision maker.

The rest of the paper is organized as follows. In Section 2 we discuss a number of known results, which we call propositions throughout the section. Those propositions lead naturally to our main result, which is formulated in Section 3 as Theorem 3.1. In Section 4 we give an illustrative example that demonstrates the sharpness of the newly established Grüss-type bound.

\section{A Discussion of Known Results}

Grüss [13] proved that if two functions $v$ and $w$ satisfy bounds $a \leq v(x) \leq A$ and $b \leq w(x) \leq$ $B$ for all $x \in\left[x_{1}, x_{2}\right]$, then

$$
\left|\frac{1}{x_{2}-x_{1}} \int_{x_{1}}^{x_{2}} v(x) w(x) d x-\frac{1}{\left(x_{2}-x_{1}\right)^{2}} \int_{x_{1}}^{x_{2}} v(x) d x \int_{x_{1}}^{x_{2}} w(x) d x\right| \leq \frac{1}{4}(A-a)(B-b) .
$$

This is known in the literature as the Grüss bound. If $X$ denotes a uniformly distributed random variable with the support $\left[x_{1}, x_{2}\right]$, then statement $(2.1)$ can be rewritten as

$$
|\operatorname{Cov}[v(X), w(X)]| \leq \frac{1}{4}(A-a)(B-b) .
$$

This is a covariance bound. If we replace $v(X)$ and $w(X)$ by two general random variables $X$ and $Y$ with supports $[a, A]$ and $[b, B]$, respectively, then from (2.2) we obtain the following covariance bound (Dragomir [14, 15]; also Zitikis [6]):

$$
|\operatorname{Cov}[X, Y]| \leq \frac{1}{4}(A-a)(B-b)
$$

We emphasize that the random variables $X$ and $Y$ in (2.3) are not necessary uniformly distributed. They are general random variables, except that we assume $X \in[a, A]$ and $Y \in[b, B]$, and no dependence structure between $X$ and $Y$ is assumed.

There are many results sharpening Grüss's bound under various bits of additional information (see, e.g., Dragomir [14, 15], and references therein). For example, Anastassiou and Papanicolaou [16] have established the following bound.

Proposition 2.1. Let $X \in[a, A]$ and $Y \in[b, B]$ be two random variables with joint density function $h$, assuming that it exists, and denote the (marginal) densities of $X$ and $Y$ by $f$ and $g$, respectively. Then

$$
|\operatorname{Cov}[X, Y]| \leq \frac{\int_{b}^{B} \int_{a}^{A}|h(x, y)-f(x) g(y)| d x d y}{4}(A-a)(B-b) .
$$


Approaching the problem from a different angle, Zitikis [6] has sharpened Grüss's bound by including restrictions on the means of the random variables $X$ and $Y$, as stated in the next proposition.

Proposition 2.2. Let $X \in[a, A]$ and $Y \in[b, B]$ be two random variables. Furthermore, let $\left[\mu_{a}, \mu_{A}\right] \subseteq[a, A]$ and $\left[\mu_{b}, \mu_{B}\right] \subseteq[b, B]$ be intervals such that $\mathbf{E}[X] \in\left[\mu_{a}, \mu_{A}\right]$ and $\mathbf{E}[Y] \in\left[\mu_{b}, \mu_{B}\right]$. Then

$$
|\operatorname{Cov}[X, Y]| \leq \frac{(1-\mathfrak{A})(1-\mathfrak{B})}{4}(A-a)(B-b),
$$

where $\mathfrak{A}$ and $\mathfrak{B}$ are "information coefficients" defined by

$$
\begin{aligned}
& \mathfrak{A}=1-\frac{2}{A-a} \sup _{x \in\left[\mu_{a}, \mu_{A}\right]} \sqrt{(A-x)(x-a)}, \\
& \mathfrak{B}=1-\frac{2}{B-b} \sup _{y \in\left[\mu_{b}, \mu_{B}\right]} \sqrt{(B-y)(y-b)} .
\end{aligned}
$$

When there is no "useful information," then the two information coefficients $\mathfrak{A}$ and $\mathfrak{B}$ are equal to 0 by definition (Zitikis [6]), and thus bound (2.5) reduces to the classical Grüss bound.

Mitrinović et al. [17] have in detail discussed Chebyshev's integral inequality, formulated next as a proposition, which gives an insight into Grüss's inequality and especially into the sign of the covariance $\operatorname{Cov}[X, Y]$.

Proposition 2.3. Let $v, w$, and $f$ be real functions defined on $\left[x_{1}, x_{2}\right]$, and let $f$ be nonnegative and integrable. If the functions $v$ and $w$ are both increasing, or both decreasing, then

$$
\int_{x_{1}}^{x_{2}} f(x) d x \times \int_{x_{1}}^{x_{2}} v(x) w(x) f(x) d x \geq \int_{x_{1}}^{x_{2}} v(x) f(x) d x \times \int_{x_{1}}^{x_{2}} w(x) f(x) d x .
$$

If, however, one of the two functions $v$ and $w$ is increasing and the other one is decreasing, then inequality (2.7) is reversed.

With an appropriately defined random variable $X$ (see a note following Grüss's inequality (2.1) above), Chebyshev's integral inequality (2.7) can be rewritten in the following form:

$$
\operatorname{Cov}[v(X), w(X)] \geq 0
$$

As we will see in a moment, inequality (2.8) is also implied by the notion of positive quadrant dependence (Lehmann [12]). For details on economic applications of Chebyshev's integral inequality (2.8), we refer to Athey [18], Wagener [19], and references therein. 
There have been many attempts to express the covariance $\operatorname{Cov}[X, Y]$ in terms of the cumulative distribution functions of the random variables $X$ and $Y$. Among them is a result by Hoeffding [20], who proved that

$$
\operatorname{Cov}[X, Y]=\iint[H(x, y)-F(x) G(y)] d x d y
$$

where $H$ is the joint cumulative distribution function of $(X, Y)$, and $F$ and $G$ are the (marginal) cumulative distribution functions of $X$ and $Y$, respectively. Mardia [21], Mardia and Thompson [22] extended Hoeffding's result by showing that

$$
\operatorname{Cov}\left[X^{r}, Y^{s}\right]=\iint[H(x, y)-F(x) G(y)] r x^{r-1} s y^{s-1} d x d y
$$

For further extensions of these results, we refer to Sen [23] and Lehmann [12]. Cuadras [24] has generalized these works by establishing the following result.

Proposition 2.4. Let $v$ and $w$ be any real functions of bounded variation and defined, respectively, on the intervals $[a, A]$ and $[b, B]$ of the extended real line $[-\infty, \infty]$. Furthermore, let $X \in[a, A]$ and $Y \in[b, B]$ be any random variables such that the expectations $\mathbf{E}[v(X)], \mathbf{E}[w(Y)]$, and $\mathbf{E}[v(X) w(Y)]$ are finite. Then

$$
\operatorname{Cov}[v(X), w(Y)]=\int_{(b, B]} \int_{(a, A]}[H(x, y)-F(x) G(y)] d v(x) d w(y)
$$

Equation (2.11) plays a crucial role in establishing our main result, which is Theorem 3.1 in the next section. To facilitate easier intuitive understanding of that section, we note that the function

$$
C(x, y)=H(x, y)-F(x) G(y)
$$

which is the integrand on the right-hand side of (2.11), governs the dependence structure between the random variables $X$ and $Y$. For example, when $C(x, y)=0$ for all $x$ and $y$, then the random variables are independent. Hence, departure of $C(x, y)$ from 0 serves a measure of dependence between $X$ and $Y$. Depending on which side (positive or negative) the departure from 0 takes place, we have positive or negative dependence between the two random variables. Specifically, when $C(x, y) \geq 0$ for all $x$ and $y$, then $X$ and $Y$ are called positively quadrant dependent, and when $C(x, y) \leq 0$ for all $x$ and $y$, then the random variables are negatively quadrant dependent. For applications of these notions of dependence and also for further references, we refer to the monographs by Balakrishnan and Lai [25], Denuit et al. [26]. 


\section{A New Grüss-Type Bound}

We start this section with a bound that plays a fundamental role in our subsequent considerations. Namely, for all $x, y \in \mathbf{R}$, we have that

$$
|C(x, y)| \leq \frac{1}{4}
$$

irrespectively of the dependence structure between the random variables $X$ and $Y$. Bound (3.1) can be verified as follows. First, for any event $A$, the probability $\mathbf{P}[A]$ is the expectation $\mathbf{E}[1\{A\}]$ of the indicator $1\{A\}$, which is a random variable taking on the value 1 if the event $A$ happens, and 0 otherwise. Hence, $C(x, y)$ is equal to the covariance $\operatorname{Cov}[1\{X \leq x\}, 1\{Y \leq y\}]$. Next we use the Cauchy-Schwarz inequality to estimate the latter covariance and thus obtain that

$$
|C(x, y)| \leq \sqrt{\operatorname{Var}[1\{X \leq x\}] \operatorname{Var}[1\{Y \leq y\}]} .
$$

Since $1\{X \leq x\}$ is a binary random variable taking on the two values 1 and 0 with the probabilities $\mathbf{P}[X \leq x]$ and $\mathbf{P}[X>x]$, respectively, the variance $\operatorname{Var}[1\{X \leq x\}]$ is equal to the product of the probabilities $\mathbf{P}[X \leq x]$ and $\mathbf{P}[X>x]$. The product does not exceed $1 / 4$. Likewise, the variance $\operatorname{Var}[1\{Y \leq y\}]$ does not exceed 1/4. From bound (3.2) we thus have bound (3.1).

To see how bound (3.1) is related to Grüss's bound, we apply it on the right-hand side of (2.11). We also assume that the functions $v$ and $w$ are right-continuous and monotonic. Note that, without loss of generality in our context, the latter monotonicity assumption can be replaced by the assumption that the two functions $v$ and $w$ are non-decreasing. Hence, we have the bound

$$
|\operatorname{Cov}[v(X), w(Y)]| \leq \frac{1}{4}[v(A)-v(a)][w(B)-w(b)]
$$

which is Grüss's bound written in a somewhat different form than that in (2.2).

The following theorem sharpens the upper bound of Grüss's covariance inequality (3.3) by utilizing the notion of quadrant dependence (cf. Lehmann [12]) and incorporating constrains on the means of random variables $X$ and $Y$ (cf. Zitikis [6]).

Theorem 3.1. Let $X \in[a, A]$ and $Y \in[b, B]$ be any random variables, and let $\mathfrak{D} \in[0,1]$, which one calls the "dependence coefficient," be such that

$$
|C(x, y)| \leq \frac{1-\mathfrak{D}}{4}
$$

for all $x \in[a, A]$ and $y \in[b, B]$. Furthermore, let $v$ and $w$ be two right-continuous and nondecreasing functions defined on $[a, A]$ and $[b, B]$, respectively, and let $\Omega_{1}$ and $\Omega_{2}$ be intervals such that $\mathbf{E}[v(X)] \in \Omega_{1} \subseteq[v(a), v(A)]$ and $\mathbf{E}[w(Y)] \in \Omega_{2} \subseteq[w(b), w(B)]$. Then

$$
|\operatorname{Cov}[v(X), w(Y)]| \leq \frac{\min \{1-\mathfrak{D},(1-\mathfrak{A})(1-\mathfrak{B})\}}{4}[v(A)-v(a)][w(B)-w(b)],
$$


where $\mathfrak{A}$ and $\mathfrak{B}$ are "information coefficients" defined by

$$
\begin{aligned}
\mathfrak{A} & =1-\frac{2}{v(A)-v(a)} \sup _{x \in \Omega_{1}} \sqrt{[v(b)-x][x-v(a)]}, \\
\mathfrak{B} & =1-\frac{2}{w(B)-w(b)} \sup _{y \in \Omega_{2}} \sqrt{[w(B)-y][y-w(b)]} .
\end{aligned}
$$

Before proving the theorem, a few clarifying notes follow. If there is no "useful information" (see Zitikis [6] for the meaning) about the location of the means $\mathrm{E}[v(X)]$ and $\mathrm{E}[w(Y)]$ inside the intervals $[v(a), v(A)]$ and $[w(b), w(B)]$, respectively, then the two information coefficients $\mathfrak{A}$ and $\mathfrak{B}$ are equal to 0 by definition, and thus $(1-\mathfrak{A})(1-\mathfrak{B})$ is equal to 1. Furthermore, if there is no "useful dependence information" between $X$ and $Y$, then $\mathfrak{D}=0$ by definition. Hence, in the presence of no "useful information" about the means and dependence, the coefficient $\min \{1-\mathfrak{D},(1-\mathfrak{A})(1-\mathfrak{B})\} / 4$ reduces to the classical Grüss coefficient $1 / 4$.

Proof of Theorem 3.1. Since $|C(x, y)| \leq(1-\mathfrak{D}) / 4$ by assumption, using (2.11) we have that

$$
\begin{aligned}
|\operatorname{Cov}[v(X), w(Y)]| & \leq \int_{(b, B]} \int_{(a, A]}|C(x, y)| d v(x) d w(y) \\
& \leq \frac{1-\mathfrak{D}}{4} \int_{(b, B]} \int_{(a, A]} d v(x) d w(y) \\
& =\frac{1-\mathfrak{D}}{4}[v(A)-v(a)][w(B)-w(b)],
\end{aligned}
$$

where the last equality holds because the functions $v$ and $w$ are right-continuous and nondecreasing. Next we restart the estimation of the covariance $\operatorname{Cov}[v(X), w(Y)]$ anew. Namely, using the Cauchy-Schwarz inequality, together with the bound

$$
\operatorname{Cov}[v(X), v(X)] \leq[v(A)-\mathrm{E}[v(X)]][\mathrm{E}[v(X)]-v(a)]
$$

and an analogous one for $\operatorname{Cov}[w(Y), w(Y)]$, we obtain that

$$
\begin{aligned}
|\operatorname{Cov}[v(X), w(Y)]| & \leq \sqrt{\operatorname{Cov}[v(X), v(X)]} \sqrt{\operatorname{Cov}[w(Y), w(Y)]} \\
& \leq \sup _{x \in \Omega_{1}} \sqrt{[v(A)-x)(x-v(a)]} \sup _{y \in \Omega_{2}} \sqrt{[w(B)-y)(y-w(b)]} \\
& =\frac{(1-\mathfrak{A})(1-\mathfrak{B})}{4}[v(A)-v(a)][w(B)-w(b)] .
\end{aligned}
$$

Combining bounds (3.7) and (3.9), we arrive at bound (3.5), thus completing the proof of Theorem 3.1. 


\section{An Example}

Here we present an example that helps to compare the bounds of Grüss [13], Zitikis [6], and the one of Theorem 3.1.

To make our considerations as simple as possible, yet meaningful, we choose to work with the functions $v(x)=x$ and $w(y)=y$, and also assume that the random variables $X$ and $Y$ take on values in the interval $[0,1]$. Grüss's bound (2.3) implies that

$$
|\operatorname{Cov}[X, Y]| \leq \frac{1}{4}=0.25
$$

Assume now that the pair $(X, Y)$ has a joint density function, $f(s, t)$, and let it be equal to $\left(s^{2}+t^{2}\right) 3 / 2$ for $s, t \in[0,1]$, and 0 for all other $s, t \in \mathbf{R}$. The random variables $X$ and $Y$ take on values in the interval $[0,1]$ as before, but we can now calculate their means and thus apply Proposition 2.2 with appropriately specified " $\mu$-constraints."

The joint cumulative distribution function $H(x, y)=\int_{0}^{y} \int_{0}^{x} f(s, t) d s d t$ of the pair $(X, Y)$ can be expressed by the formula $H(x, y)=x y\left(x^{2}+y^{2}\right) / 2$. Thus, the (marginal) cumulative distribution functions of $X$ and $Y$ are equal to $F(x)=H(x, 1)=x\left(x^{2}+1\right) / 2$ for all $x \in[0,1]$ and $G(y)=H(1, y)=y\left(y^{2}+1\right) / 2$ for all $y \in[0,1]$, respectively. Using the equation $\mathbf{E}[X]=$ $\int_{0}^{1}(1-F(x)) d x$, we check that $\mathrm{E}[\mathrm{X}]=5 / 8$. Likewise, we have $\mathrm{E}[Y]=5 / 8$. Consequently, we may let the $\mu$-constraints on the means $\mathbf{E}[X]$ and $\mathbf{E}[Y]$ be as follows: $\mu_{a}=5 / 8=\mu_{A}$ and $\mu_{b}=5 / 8=\mu_{B}$. We also have $a=0=b$ and $A=1=B$, because [0,1] is the support of the two random variables $X$ and $Y$. These notes and the definitions of $\mathfrak{A}$ and $\mathfrak{B}$ given in Proposition 2.2 imply that $1-\mathfrak{A}=1-\mathfrak{B}=\sqrt{15 / 16}$. Consequently, bound (2.5) implies that

$$
|\operatorname{Cov}[X, Y]| \leq \frac{15}{64}=0.2344
$$

which is an improvement upon bound (4.1), and thus upon (4.2).

We next utilize the dependence structure between $X$ and $Y$ in order to further improve upon bound (4.2). With $\mathfrak{A}$ and $\mathfrak{B}$ already calculated, we next calculate $\mathfrak{D}$. For this, we use the above formulas for the three cumulative distribution functions and see that $C(x, y)=$ $x y\left(x^{2}-1\right)\left(1-y^{2}\right) / 4$. (The negative sign of $C(x, y)$ for all $x, y \in(0,1)$ reveals that the random variables $X$ and $Y$ are negatively quadrant dependent.) Furthermore, we check that $|C(x, y)|$ attains its maximum at the point $(1 / \sqrt{3}, 1 / \sqrt{3})$. Hence, the smallest upper bound for $|C(x, y)|$ is $1 / 27$, and so we have $1-\mathfrak{D}=4 / 27$, which is less than $(1-\mathfrak{A})(1-\mathfrak{B})=15 / 16$. Hence, bound (3.5) implies that

$$
|\operatorname{Cov}[X, Y]| \leq \frac{1}{27}=0.0370
$$

which is a considerable improvement upon bounds (4.1) and (4.2).

We conclude this example by noting that the true value of the covariance $\operatorname{Cov}[X, Y]$ is

$$
\operatorname{Cov}[X, Y]=-\frac{1}{64}=-0.0156
$$


which we have calculated using the equation $\operatorname{Cov}[X, Y]=\int_{0}^{1} \int_{0}^{1} C(x, y) d x d y$ (cf. (2.9)) and the above given expression for $C(x, y)$.

\section{Acknowledgments}

The authors are indebted to four anonymous referees, the editor in charge of the manuscript, Soo Hak Sung, and the Editor-in-Chief, Ravi P. Agarwal, for their constructive criticism and numerous suggestions that have resulted in a considerable improvement of the paper. The third author would also like to thank Robert B. Miller and Howard E. Thompson for their continuous guidance and encouragement. The research has been partially supported by grants from the University of Montevideo, University of Coruña, Hong Kong Baptist University, and the Natural Sciences and Engineering Research Council (NSERC) of Canada.

\section{References}

[1] M. Egozcue, L. Fuentes Garcia, and W.-K. Wong, "On some covariance inequalities for monotonic and non-monotonic functions," Journal of Inequalities in Pure and Applied Mathematics, vol. 10, no. 3, article 75, pp. 1-7, 2009

[2] E. Furman and R. Zitikis, "Weighted premium calculation principles," Insurance: Mathematics and Economics, vol. 42, no. 1, pp. 459-465, 2008.

[3] E. Furman and R. Zitikis, "Weighted risk capital allocations," Insurance: Mathematics and Economics, vol. 43, no. 2, pp. 263-269, 2008.

[4] E. Furman and R. Zitikis, "Weighted pricing functionals with applications to insurance: an overview," North American Actuarial Journal, vol. 13, pp. 483-496, 2009.

[5] E. Furman and R. Zitikis, "General Stein-type covariance decompositions with applications to insurance and Finance," to appear in ASTIN Bulletin-The Journal of the International Actuarial Association.

[6] R. Zitikis, "Grüss's inequality, its probabilistic interpretation, and a sharper bound," Journal of Mathematical Inequalities, vol. 3, no. 1, pp. 15-20, 2009.

[7] S. S. Dragomir, Advances in Inequalities of the Schwarz, Grüss and Bessel Type in Inner Product Spaces, Nova Science, New York, NY, USA, 2005.

[8] S. S. Dragomir and R. P. Agarwal, "Some inequalities and their application for estimating the moments of guessing mappings," Mathematical and Computer Modelling, vol. 34, no. 3-4, pp. 441-468, 2001.

[9] S. S. Dragomir and N. T. Diamond, "A discrete Grüss type inequality and applications for the moments of random variables and guessing mappings," in Stochastic Analysis and Applications, vol. 3, pp. 21-35, Nova Science, New York, NY, USA, 2003.

[10] S. Izumino and J. E. Pečarić, "Some extensions of Grüss' inequality and its applications," Nihonkai Mathematical Journal, vol. 13, no. 2, pp. 159-166, 2002.

[11] S. Izumino, J. E. Pečarić, and B. Tepeš, "A Grüss-type inequality and its applications," Journal of Inequalities and Applications, vol. 2005, no. 3, pp. 277-288, 2005.

[12] E. L. Lehmann, "Some concepts of dependence," Annals of Mathematical Statistics, vol. 37, pp. 11371153, 1966.

[13] G. Grüss, “Über das maximum des absoluten betrages von $1 /(b-a) \int_{a}^{b} f(x) g(x) d x-$ $1 /(b-a)^{2} \int_{a}^{b} f(x) d x \int_{a}^{b} g(x) d x, "$ Mathematische Zeitschrift, vol. 39, no. 1, pp. 215-226, 1935.

[14] S. S. Dragomir, "A generalization of Grüss's inequality in inner product spaces and applications," Journal of Mathematical Analysis and Applications, vol. 237, no. 1, pp. 74-82, 1999.

[15] S. S. Dragomir, "New inequalities of the Kantorovich type for bounded linear operators in Hilbert spaces," Linear Algebra and Its Applications, vol. 428, no. 11-12, pp. 2750-2760, 2008.

[16] G. A. Anastassiou and V. G. Papanicolaou, "Probabilistic inequalities and remarks," Applied Mathematics Letters, vol. 15, no. 2, pp. 153-157, 2002.

[17] D. S. Mitrinović, J. E. Pečarić, and A. M. Fink, Classical and New Inequalities in Analysis, vol. 61 of Mathematics and Its Applications (East European Series), Kluwer Academic Publishers, Dordrecht, The Netherlands, 1993. 
[18] S. Athey, "Monotone comparative statics under uncertainty," Quarterly Journal of Economics, vol. 117, no. 1, pp. 187-223, 2002.

[19] A. Wagener, "Chebyshev's algebraic inequality and comparative statics under uncertainty," Mathematical Social Sciences, vol. 52, no. 2, pp. 217-221, 2006.

[20] W. Hoeffding, "Masstabinvariante korrelationstheorie," in Schriften des Matematischen Instituts für Angewandte Matematik der Universität Berlin, vol. 5, pp. 179-233, 1940.

[21] K. V. Mardia, "Some contributions to contingency-type bivariate distributions," Biometrika, vol. 54, pp. 235-249, 1967.

[22] K. V. Mardia and J. W. Thompson, “Unified treatment of moment-formulae," Sankhyā Series A, vol. 34, pp. 121-132, 1972.

[23] P. K. Sen, "The impact of Wassily Hoeffding's research on nonparametric," in Collected Works of Wassily Hoeffding, N. I. Fisher and P. K. Sen, Eds., pp. 29-55, Springer, New York, NY, USA, 1994.

[24] C. M. Cuadras, "On the covariance between functions," Journal of Multivariate Analysis, vol. 81, no. 1, pp. 19-27, 2002.

[25] N. Balakrishnan and C. D. Lai, Continuous Bivariate Distributions, Springer, New York, NY, USA, 2nd edition, 2009.

[26] M. Denuit, J. Dhaene, M. Goovaerts, and R. Kaas, Actuarial Theory for Dependent Risks: Measures, Orders and Models, John Wiley \& Sons, Chichester, UK, 2005. 